\title{
THE LENGTH SPECTRUM OF A RIEMANN SURFACE IS ALWAYS OF UNBOUNDED MULTIPLICITY
}

\author{
BURTON RANDOL
}

\begin{abstract}
I show that the length spectrum of a Riemann surface is always of unbounded multiplicity, and indicate connections with recent work of Guillemin and Kazhdan.
\end{abstract}

V. Guillemin and D. Kazhdan have recently established [4] that if $S$ is a compact surface having a metric of negative curvature, then under rather general conditions, the spectrum of the Schrödinger operator $\Delta+q(x)$ on $S$ determines the function $q(x)$. The major hypothesis of their theorem is that the length spectrum of $S$ be simple, i.e., no two distinct unoriented closed geodesics on $S$ have the same length. It is known (cf. [1]) that this is true for most metrics. On the other hand, it is a curious fact that it is never true in the Riemann surface case, i.e., when $S$ has constant curvature -1 . In this note, we will show this and somewhat more, namely that in the constant curvature case, the length spectrum is never even of bounded multiplicity. I am told that in the same vein, T. Jørgensen has observed, using a geometric argument, that there are always present lengths of multiplicity at least 2.

We now pass to a proof of the result.

It follows from a result of $\mathrm{R}$. Horowitz [5, p. 648], that if $A$ and $B$ are two elements of $\operatorname{PSL}(2, R)$ which generate a free group $G$, then for any positive integer $N$, there exist words $W_{1}, \ldots, W_{N}$ in $A$ and $B$ such that:

(1) None of the $W_{1}, \ldots, W_{N}$ are conjugate in $G$.

(2) No two of the $W_{1}, \ldots, W_{N}$ are inverses of one another.

(3) $\left|\operatorname{tr} W_{1}\right|=\left|\operatorname{tr} W_{2}\right|=\cdots=\left|\operatorname{tr} W_{N}\right|$.

Now the conjugacy problem for fundamental groups of compact orientable surfaces was solved in 1912 by Dehn [2]. Cf. also [3]. It is an immediate consequence of Dehn's paper (p. 420) that if $a_{1}, b_{1}, a_{2}, b_{2}, \ldots, a_{g}, b_{g}$, $\left(a_{1} b_{1} a_{1}^{-1} b_{1}^{-1}\right) \cdots\left(a_{g} b_{g} a_{g}^{-1} b_{g}^{-1}\right)=1$, is a standard presentation of the fundamental group $P$ of a surface of genus $g>1$, and if $x$ and $y$ are two elements in the free group $F$ generated by $a_{1}, \ldots, a_{g}$, then $x$ and $y$ are conjugate in $P$ if and only if they are conjugate in $F$. Thus, if in the Horowitz example we take $x=a_{1}, y=a_{2}$, we immediately find, using the correspondence between matrices and geodesics, that the multiplicity of the length spectrum of a Riemann surface is never bounded. The same technique applies to the noncompact case, since the fundamental group is then a free group.

Received by the editors May 16, 1979.

AMS (MOS) subject classifications (1970). Primary 30A46.

() 1980 American Mathematical Society 0002-9939/80/0000-0135/\$01.50 


\section{REFERENCES}

1. R. Abraham, Bumpy metrics, Global Analysis, Proc. Sympos. Pure Math., vol. 14, Amer. Math. Soc., Providence, R. I., 1970, pp. 1-3.

2. M. Dehn, Transformation der Kurven auf zweiseitigen Flächen, Math. Ann. 72 (1912), 413-421.

3. M. Greendlinger, On Dehn's algorithms for the conjugacy and word problems, with applications, Comm. Pure Appl. Math. 13 (1960), 641-677.

4. V. Guillemin and D. Kazhdan, Some inverse spectral results for negatively curved two-manifolds (preprint).

5. R. Horowitz, Characters of free groups represented in the two-dimensional special linear group, Comm. Pure Appl. Math. 25 (1972), 635-649.

Department of Mathematics, Graduate Center, City University of New York, New York, NEW YORK 10036 